\title{
Traduire
}

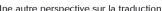

Revue française de la traduction

$243 \mid 2020$

Les arts du spectacle

\section{Cantata greca ou le désir de traduire. Moni Ovadia joue Yannis Ritsos}

\section{Chiara Gallo}

\section{Q OpenEdition \\ 1 Journals}

\section{Édition électronique}

URL : http://journals.openedition.org/traduire/2163

DOI : 10.4000/traduire.2163

ISSN : 2272-9992

Éditeur

Société française des traducteurs

Édition imprimée

Date de publication : 15 décembre 2020

Pagination : 59-64

ISSN : 0395-773X

\section{Référence électronique}

Chiara Gallo, «Cantata greca ou le désir de traduire. Moni Ovadia joue Yannis Ritsos », Traduire [En ligne], 243 | 2020, mis en ligne le 15 décembre 2020, consulté le 31 décembre 2020. URL : http:// journals.openedition.org/traduire/2163 ; DOI : https://doi.org/10.4000/traduire.2163 


\title{
Cantata greca ou le désir de traduire
}

\author{
Moni Ovadia joue Yannis Ritsos
}

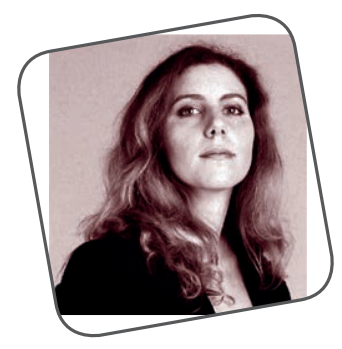

\section{Chiara Gallo}

Néanmoins, au-dessus de tout ce bruit, du désordre, des traductions / tu as l'impression qu'il reste intact le cri silencieux de l'Intraduisible / inouï, profond, menaçant, lointain, étranger, / familier pourtant; - et ressurgit en toi / le désir de traduire'.

Créé en 2012 au théâtre Cavallerizza de Reggio d’Émilie (Italie), le spectacle Cantata greca est avant tout la rencontre virtuelle de deux hommes qui se ressemblent par leur façon particulière de se tenir face au monde et avec le monde, dans une même quête du «radicalement humain»: le poète et résistant communiste grec Yannis Ritsos (1909-1990), chantre de la grécité meurtrie et pourtant florissante, et Moni Ovadia, artiste bulgaro-italien de culture juive, passionnément attaché à la tradition yiddish d'Europe centrale, mais aussi personnage très présent (et médiatisé) dans les mouvements citoyens en Italie. 
Profondément touché par l'œuvre et le parcours humain du poète grec, dans lesquels il retrouve les enjeux qui animent sa propre recherche artistique et son activité politique, Moni Ovadia entame, à partir de 1990, un travail de longue haleine sur la poésie de Yannis Ritsos, dont Cantata greca est l'expression la plus accomplie.

Le spectacle se compose de deux monologues poétiques en vers libres, Delfi et La sonata al chiaro di luna ${ }^{2}$, indépendants mais construits de façon similaire autour de la question du temps, de la liberté ou de l'éblouissante vanité des choses. Dans le premier, un guide touristique âgé du site de Delphes évoque avec lassitude son inutile routine quotidienne, ses récits que personne n'écoute, les touristes qui traversent le site sans en voir la beauté; dans La Sonate au clair de lune, une vieille dame en noir implore son jeune visiteur de lui permettre de l'accompagner dans la cité, lieu tant rêvé de la vie et des échanges humains, pour finalement renoncer.

Dépourvus d'action, ces deux monologues reposent uniquement sur la parole, dont l'importance se retrouve amplifiée dans Cantata greca, construit sur l'alternance et le mélange, à parts égales, de l'italien et du grec. Chez Moni Ovadia, le "désir de traduire», que nombre d'artistes évoquent au sens figuré comme étant à l'origine du processus de création, se concrétise au sens propre sur le plateau et sous-tend les principaux choix de mise en scène; l'acte de traduire, exploité dans toute sa capacité performative, est aussi investi d'une forte portée éthique et politique, en cohérence avec le profil de l'interprète.

Le rôle structural de la traduction dans Cantata greca se manifeste de deux façons: d'abord, le passage de l'italien au grec coïncide avec un changement radical dans le jeu de Moni Ovadia, donc dans la lecture qu'il fait du texte ritsien; en second lieu, les deux langues et leurs relatives fonctions dramaturgiques sont prises à leur tour dans un réseau de correspondances avec d'autres langages scéniques tels que le chant ou la danse.

2. Par la suite, les titres seront donnés en français pour une meilleure lisibilité: Delphes et La Sonate au clair de lune. 
Généralement, un même passage est joué dans les deux langues, en commençant indifféremment par l'une ou l'autre. En grec, Moni Ovadia met l'accent sur la dimension quotidienne et immédiate de l'écriture de Yannis Ritsos, en donnant une interprétation réaliste et parfois comique de ses personnages, tandis qu'en italien, il reprend la posture du conteur ou celle du poète lui-même. Par moments, la traduction est incomplète, sélective, de façon à faire ressortir certaines images du texte. À ce propos, un passage de Delphes est particulièrement significatif, quand le protagoniste exprime toute sa lassitude de devoir raconter chaque jour les mêmes batailles de l'Antiquité: Moni Ovadia, intéressé par les échos contemporains du texte, joue uniquement en grec, de façon très théâtrale, cette interminable liste de tueries anciennes, en donnant à voir, au spectateur non grécophone, un personnage plutôt drôle, excédé par la tâche répétitive qui lui est imposée. Mais cette tirade en grec est entrecoupée par des digressions en italien d'une tout autre teneur: avec une parole droite et sobre, Moni Ovadia appuie les réflexions et les images les plus sombres; car ce sont «toujours les mêmes maudites guerres» et «les statues ont caché leurs yeux derrière leurs mains» pour ne pas voir "les morts qui aveuglent les yeux». Cette stratégie s'hybride à la fin du passage, lorsqu'est crié à trois fois un mot grec que même un

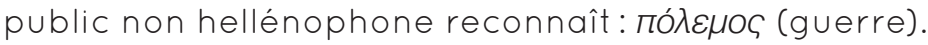

Au-delà de solutions comme celle-ci, liées au message antimilitariste que Moni Ovadia tient à exprimer, l'aspect le plus intéressant de ce bilinguisme, et peut-être aussi le plus profondément politique, c'est le plaisir évident que l'artiste prend à jover en grec et avec le grec (qu'il a appris "grâce à Yannis Ritsos»), à s'approprier la langue de l'autre, à la reproduire en la regardant avec la distance infime qui le sépare d'elle. II n'y a, dans Cantata greca, aucun rapport hiérarchique entre les deux langues, si bien qu'à la fin, le public ne sait plus si c'est l'italien qui traduit le grec ou inversement. De plus, cette parité de traitement invite le spectateur à endosser lui aussi le rôle de l'apprenti traducteur, à tenter d'imaginer des correspondances, de reconnaitre des modulations récurrentes, de se familiariser avec les rythmes de la langue grecque. S'il éprouve à son tour le "désir de traduire», le spectateur peut également faire appel aux suggestions venant d'autres 
langages scéniques, notamment le chant et la danse qui, comme c'est souvent le cas dans les spectacles de Moni Ovadia, occupent une place importante dans ce diptyque.

Plongé dans une ambiance sonore composée par Piero Milesi, Delphes passe avec une grande souplesse du théâtre au concert et inversement, et c'est souvent au grec que Moni Ovadia confie les moments les plus lyriques de sa performance, pour reprendre ensuite les mêmes passages en italien, tout en revenant à la parole dite. Comme dans le cas cité précédemment, certaines variations permettent de mettre en valeur des images ou des mots-clés du texte de Yannis Ritsos, tout en offrant des repères traductifs au public. L'un des nœuds dramatiques de Delphes, par exemple, est une longue réflexion sur la liberté, que Moni Ovadia choisit de présenter d'abord en grec, sous forme de chant, avec une modulation particulière et immédiatement reconnaissable

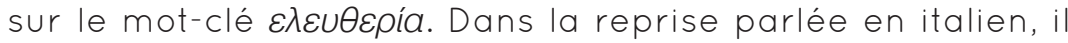
revient soudain au chant sur le seul mot libertà, en reproduisant cette même modulation.

Dans La Sonate au clair de lune, organisée, comme le titre l'indique, autour du premier mouvement de la sonate éponyme de Beethoven, Moni Ovadia travaille principalement sur les rythmes et les temps de la parole, qui reproduisent ceux de la musique. À sa figure de conteur/poète correspond ici un double féminin, la danseuse Ornella Balestra, qui évolve sur un plateau baignant dans une obscurité permanente, dans une torpeur gluante; son corps est toujours fragmenté - des mains, une jambe, parfois le visage. Ici, la danse assume une fonction traductive similaire à celle du chant dans Delphes; les correspondances qu'elle suggère sont pourtant moins linéaires, moins immédiatement lisibles. Par exemple, dans un passage crucial du texte, Yannis Ritsos introduit l'image d'une vieille ourse de foire qui, lasse de se produire dans un spectacle cruel et humiliant, décide de s'allonger par terre et de laisser les passants lui marcher dessus, dans un renoncement libératoire; mais bientôt, la nouvelle souffrance est pire que l'ancienne, et l'ourse se remet sur ses pattes et recommence son numéro. Pendant que Moni Ovadia jove ce morceau en grec, la danseuse esquisse des bribes d'images, quelques gestes menus et indéchiffrables sur le moment; par la suite, en écoutant le même passage en italien, durant 
lequel la danseuse reste complètement immobile, le public reconnaîtra peut-être les mimiques de l'ourse.

Cette traduction tardive, parfois incertaine et toujours incomplète que le spectateur est amené à produire au cours du spectacle, révèle la finesse de la lecture que Moni Ovadia fait de la poésie ritsienne. Cantata greca est pensé pour un public italien non hellénophone; contrairement à ce que l'on pourrait imaginer, connaître le grec n'est pas un avantage. En effet, le spectateur hellénophone, en ayant immédiatement accès au sens, risque de ne pas pouvoir s'abandonner au mystère dans lequel plonge, pendant un temps, le reste de la salle. Or ce sas d'incompréhension est non seulement un moment essentiel de l'expérience que Moni Ovadia propose à son public, mais il reflète aussi un trait majeur de l'œuvre de Yannis Ritsos: si d'une part, comme il le dit dans des vers célèbres, le poète recherche une expression toujours plus simple et précise, pour pouvoir enfin «appeler un chat un chat», il sait aussi se rendre devant ce qu'il appelle "l'intraduisible». Pareillement, l'effort traductif autour duquel Cantata greca est organisé ne vise pas la compréhension parfaite des différents langages du diptyque, mais tente plutôt de susciter chez le spectateur une disponibilité à l'écoute et à l'accueil de ce qu'il ne comprend pas, condition nécessaire pour qu'il sente naître en lui le "désir de traduire».

Si Moni Ovadia fait de l'acte de traduire l'axe dramaturgique principal de son spectacle, il n'est pas l'auteur des versions italiennes qu'il utilise. Ce mérite revient à Nicola Crocetti, traducteur et éditeur historique de Yannis Ritsos en Italie, ainsi qu'ami intime du poète, qu'il rencontre pour la première fois, de manière rocambolesque, lorsque celui-ci est placé sous surveillance par le régime des colonels (1967-1974). Fils d'Italiens en Grèce pendant son enfance, puis exilé grec en Italie à la fin de la Seconde Guerre mondiale, Nicola Crocetti fait lui-même l'expérience de la discrimination; ce vécu le rapproche non seulement de Yannis Ritsos, mais aussi de Moni Ovadia, auquel le lie une profonde amitié. Si, dans ses nombreuses initiatives autour de la poésie ritsienne, l'acteur ne manque jamais de souligner le rôle indispensable jové par Nicola Crocetti et même d'illustrer sa stratégie traductive, ce dernier sait être à l'écoute de la lecture particulière de l'artiste. C'est ainsi que la création de Cantata greca coïncide 
avec la publication, par Nicola Crocetti, d'un petit volume réunissant Delphes et La Sonate au clair de lune, que Moni Ovadia présente dans une courte préface intitulée significativement "Yannis Ritsos - L'humain, le poétique»; les mots passionnés qu'il réserve à cette poésie résument tout aussi bien le sens le plus profond de la traduction telle qu'il l'utilise dans Cantata greca:

[...] le kaléidoscope de la dignité et de la fragilité humaine y est décliné dans toutes ses manifestations [...], sans moralismes ni jugement, avec un regard poétique qui [...] les reconnait et les montre pour qu'elles soient accueillies en nous, dans [...] les limites de ce que nous sommes.

\section{chiara.gallo@inalco.fr}

Chiara Gallo a travaillé comme traductrice et metteuse en scène. Elle est actuellement doctorante contractuelle à I'Institut national des langues et civilisations orientales (Inalco), où elle prépare une thèse intitulée Yannis Ritsos dramaturge: présence et absence d'une œuvre théâtrale méconnue, sous la direction de Stéphane Sawas. Elle est également chargée de cours sur la littérature néohellénique et les arts du spectacle en Grèce.

SOURCES CITÉES

OVADIA Moni, Cantata greca: Delfi, Reggio Emilia (Italie) : Teatro Cavallerizza, 2012 https://www.youtube.com/watch?v=YyNBF3ZdG7c, consulté le 09/11/2020.

OVADIA Moni, Cantata greca: La sonata al chiaro di luna, Reggio Emilia (Italie): Teatro Cavallerizza, 2012, https://www.youtube.com/watch?v=S1u9Bj9M9X4, consulté le 09/11/2020.

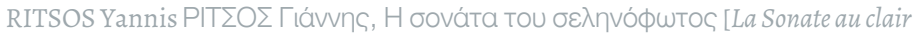

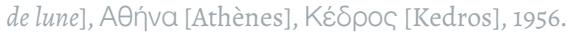

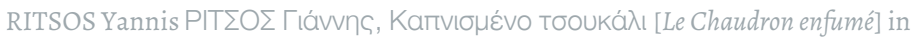

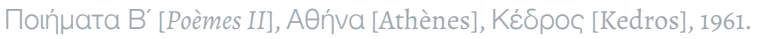

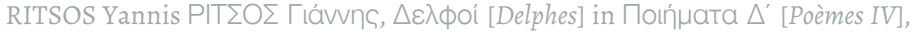

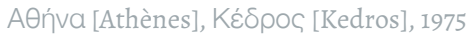

RITSOS Yannis, Delfi. La sonata al chiaro di luna, Milan (Italie), Crocetti, 2012. 